\title{
The Effect of Torsion Deformity and Medial Knee Osteoarthritis on Lower Limb Extensor Moments during Gait
}

\author{
David S. Mandeville ${ }^{1,2}$, Kenneth A. Krackow ${ }^{3,4}$, Sridhar R. Rachala ${ }^{3,4}$, Mary Bayers-Thering ${ }^{3}$ \\ ${ }^{1}$ Kinesiology and Health Science Department, California State University, Sacramento, USA; ${ }^{2}$ Department of Exercise and Nutrition \\ Sciences, State University of New York at Buffalo, Buffalo, USA; ${ }^{3}$ Department of Orthopaedic Surgery, Kaleida Health/Buffalo \\ General Hospital, Buffalo, USA; ${ }^{4}$ Department of Orthopaedics, State University of New York at Buffalo, Buffalo, USA. \\ Email: dmandeville@csus.edu
}

Received February $13^{\text {th }}, 2013$; revised March $20^{\text {th }}, 2013$; accepted March $27^{\text {th }}, 2013$

Copyright (C) 2013 David S. Mandeville et al. This is an open access article distributed under the Creative Commons Attribution License, which permits unrestricted use, distribution, and reproduction in any medium, provided the original work is properly cited.

\begin{abstract}
The aim of this study was to quantify the effect of torsion deformity on the lower limb kinetics during the loading response phase of gait. A total of 24 subjects: 6 end-staged medial knee OA with torsion deformity (TKO), 8 without torsion deformity (KOA), and 10 controls (CON) were imaged using computed tomography (CT). Internal moment of support and sagittal hip, knee and ankle joint moments were assessed using gait analysis. TKO showed greater external rotations of the proximal tibia and the distal femur compared to subjects with medial knee OA without torsion deformity and controls. TKO showed greater moment of support and a greater knee extensor moment when compared to controls when supporting the weight of the body during gait. The TKO intorsion deformity occurred as a result of a proximal malrotation of the tibia. In the presence of torsion deformity, the kinetic synergy of the lower limb showed increased total moment of support for subjects with medial knee OA. The greater extensor output from TKO may be the result of an increased muscular response to overcome an interrupted inter-segmental exchange of accelerations during the loading response phase of gait.
\end{abstract}

Keywords: Knee Osteoarthritis; Tibial Torsion; Computed Tomography; Gait Analysis; Total Moment of Support

\section{Introduction}

Krackow et al. (1990) described an internal tibial torsion deformity concomitant with flexion contracture for knee osteoarthritis which was masked as genu varum on standing radiographs and was possibly a pre-degeneration deformity [1,2]. Additionally, Krackow et al. (1997) described the impact of this abnormal bony geometry on the soft tissue attachment sites, specifically regarding a compensatory external rotation of the femoral condyles [3]. The externally rotated knee, in the presence of internal tibial torsion, was hypothesized to represent a morphological compensation in select subjects to bring the toes into a normal foot progression angle during gait.

Krackow et al. (2011) reported a test of this hypothesis using computed tomography and gait analysis for subjects with medial knee OA and apparent tibial intorsion compared to those with medial knee OA without the torsion deformity and controls. Subjects with medial knee $\mathrm{OA}$ and tibial intorsion deformity walked with a greater internal knee varus moment which was found to be predicted best by the mechanical axis and the foot progression angle, but not by the degree of tibial intorsion [4]. Additionally, in a post-hoc analysis of CT torsion values, subjects with tibial intorson and medial knee OA were found to have greater external rotation of the knee and these subjects walked with no difference of foot progression angle. However, the effect of the rotated soft tissue attachment sites of both the tibial intorsion deformity and the compensatory external rotation of the knee on the extensor moment profile for these subjects was not reported.

Weidow et al. (2005) studied radiographic coronal morphology of the hip region for knee OA subjects and reported reduced hip abductor moment arm for lateral $(22 \%)$ and for medial $(8 \%)$ knee OA subjects compared to controls [5]. Using a musculoskeletal model derived from the gait patterns of 10 healthy children, Hicks et al. (2007) found that simulated external torsion of the distal 
tibia greater than $42^{\circ}$ created a $10 \%$ decrease in the capacity of gluteus maximus, posterior gluteus medius and the soleus to extend the hip and the knee during the single limb stance of gait [6]. The diminished extensor capacity in the presence of external tibial torsion was found to be due to altered inter-segmental kinetics. The authors reported the capacity of these extensor muscles using an optimization scheme based on normative youth gait data and simulated external torsion deformity only at the distal tibia, yet the question remains, how does tibial intorsion and a compensatory externally rotated knee alter the extensor moment profile of adults with knee OA?

Extensor moment values of the lower limb joints have previously been isolated during the first peak vertical ground reaction force (vGRF) and summed to describe the lower limb extensor synergy during the loading response phase for subjects with medial knee OA [7]. However, this technique has not been used to characterize the effect of tibial intorsion deformity and medial knee OA on the lower limb's extensor moment profile. The purpose of this study was to quantify the effect of torsion deformity on the lower limb extensor moment profile during single limb support for subjects with medial knee OA and intorsion deformity compared to medial knee OA without the torsion deformity and healthy controls. We hypothesized that subjects with medial knee OA and intorsion deformity would exhibit less relative extensor moment output when supporting the body due to diminished mechanical advantage of the hip, knee and ankle extensors.

\section{Method}

\subsection{Subjects}

Twenty-four subjects were recruited into 3 groups: end-staged medial knee OA with torsion deformity (TKO, $\mathrm{n}=6, \mathrm{BMI}=34.2 \pm 3 \mathrm{~kg} / \mathrm{m}^{2}$, age $=61.8 \pm 8 \mathrm{yrs}$.); without the torsion deformity (KOA, $\mathrm{n}=8, \mathrm{BMI}=33.8 \pm 7$ $\mathrm{kg} / \mathrm{m}^{2}$, age $=59 \pm 11 \mathrm{yrs}$.) and healthy controls (CON, $\mathrm{n}$ $=10, \mathrm{BMI}=28.4 \pm 4 \mathrm{~kg} / \mathrm{m}^{2}$, age $=62.5 \pm 4$ yrs.). Apparent torsion deformity was identified using a long standing lower extremity (LSLE) radiograph. The LSLE was used to classify knee OA disease severity for all subjects based on the Kellgren/Lawrence $(\mathrm{K} / \mathrm{L})$ scale; knee OA scores were $\geq 3$ and controls were $<2$. Inclusion criteria included: age $>55$ years and the absence of self report knee pain and dysfunction. Exclusion criteria for all subjects were: rheumatoid arthritis, prior corrective surgery on the study limb, prior fracture to the femur or tibia of the study limb and current musculoskeletal trauma. This study was approved by the Institutional Review Board and all subjects provided informed, voluntary consent prior to testing.

\subsection{Computed Tomography Imaging}

A previously described partially loaded-limb CT scan protocol was used to replicate the tibiofemoral joint angle $\left(10^{\circ}\right.$ flexion) during the stance phase of gait [4]. Cross sections of the femur and the tibia were obtained proximally and distally at $3 \mathrm{~mm}$ intervals. Sections were taken from three regions: A) proximal to the femoroacetabular joint and extending to the proximal femoral shaft; B) proximal to the tibiofemoral joint and extending to the tibial tubercle; and C) proximal to the malleoli and extending to the base plate (Figure 1). Digital CT images were archived and measured by two evaluators (SRR and DSM) using a PC workstation.

The slices used to identify the proximal and distal long bone rotation angles were determined using the following protocol which has not been previously reported. The

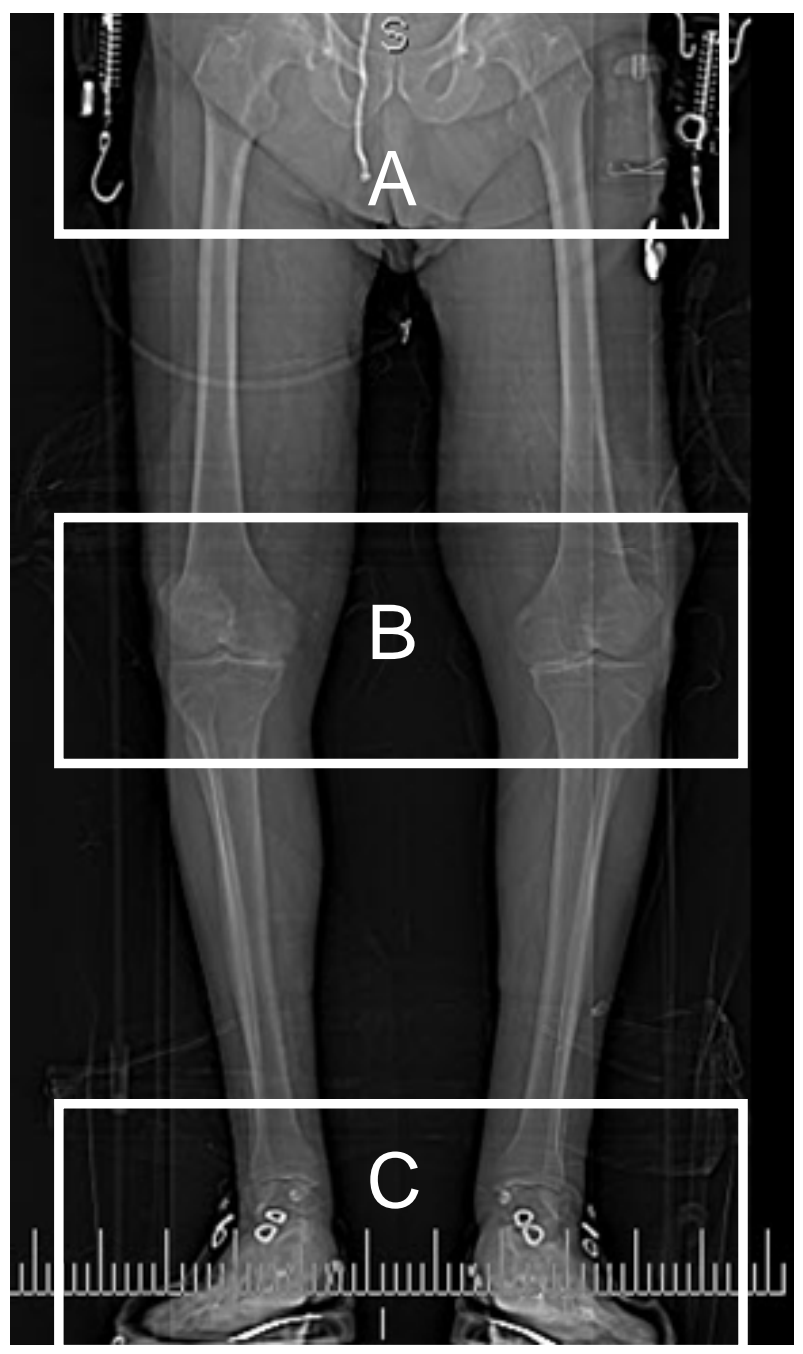

Figure 1. A computed tomography scout view of a subject with medial knee $\mathrm{OA}$ and torsion deformity showing the areas where $3 \mathrm{~mm}$ slices were used to measure lower limb torsion: the femoroacetabular region (A), the tibiofemoral region (B), and the talocrural region (C). The subject was supine with the knees supported in $10^{\circ}$ of flexion, the feet were fixed in the level walking foot progression angle while loaded axially with approximately $55 \mathrm{~N}$. 
proximal femur (PF) angle was isolated at the section which had the thickest femoral neck and was determined from the bisector of the center of the femoral head to the greater trochanter. The distal femur (DF) angle was isolated at the section with the most posterior protrusion of the femoral condyles and was determined from the bisector connecting the posterior portions of the medial and lateral femoral condyles. The proximal tibia (PT) angle was isolated at the section just above the fibular head and was determined from the midpoint bisector of the medial and lateral compartment using anterior/posterior transecting lines. The distal tibia (DT) angle was isolated at the section just superior to the talar dome and was determined from the bisector of the malleoli. All angles are relative to the horizontal axis, with external rotation values as positive. The PF, DF, PT and DT values have not been reported and will be included to add to the known long bone torsion values for both knee OA and aged-matched control subjects.

\subsection{Internal Joint Moments-Gait Analysis}

Sagittal internal net joint moments were determined using level walking motion analysis involving two force plates (Kistler Instrument Corp., Amherst, NY) and an eight-camera VICON MX20 motion capture system (Oxford Metrics, UK). A modified Cleveland Clinic set of retroreflective markers (diameter $=13 \mathrm{~mm}$ ) was used to define a 7 segment model of the pelvis and both lower extremities with a functional hip joint center estimation (Figure 2). A Newton/Euler inverse dynamic analysis was used to calculate the internal hip, knee, and ankle joint moments (C-Motion Inc., Maryland). These net joint moments (normalized to $\%$ body weight $\times$ body height)

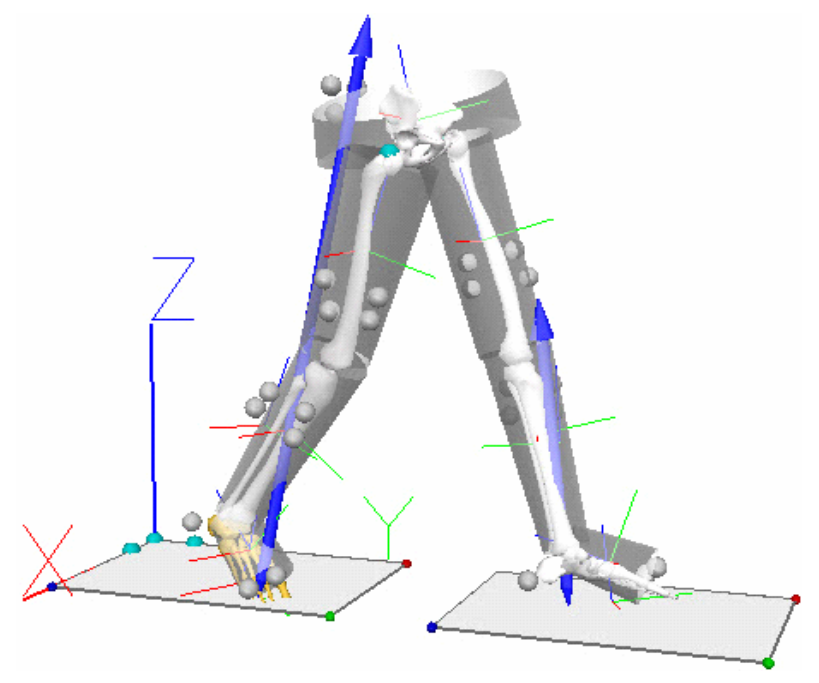

Figure 2. The seven segment model of the lower limbs (using the Cleveland Clinic marker set and gait analysis) which was used to quantify the sagittal kinetic synergy to support the body on a single limb during walking. were extracted at the first peak vertical ground reaction force (vGRF) and summed to present the total moment of support (MS) [8]. Isolating the internal joint moment values at the first peak vGRF allows for a kinetic description corresponding to the locomotor task of single limb support of the body where extensor contributions from all 3 lower limb joints are present [9]. Thus, this kinetic analysis allows a characterization of the central nervous system's mechanical output when resisting collapse of the limb during dynamic support of the body in the presence of medial knee OA and intorsion deformity.

\subsection{Data Analysis}

Between-group differences for the CT measures were assessed using one-way analyses of variance $(\alpha<0.05$, SPSS, Chicago, Illinois). Analysis of co-variance using gait velocity as a covariate was used to test for betweengroup differences for the moment of support, the hip, knee, and ankle joint moments $(\alpha<0.05$, SPSS, Chicago, Illinois). Post-hoc contrasts were used to test for between-group differences in the adjusted mean kinetic values.

\section{Results}

Subjects with medial knee OA and tibial intorsion showed significantly greater external rotation at the distal femur when compared to both KOA and CON (Table 1). Additionally, TKO had significantly greater external rotation of the proximal tibia when compared to both KOA and CON (Table 1). The proximal femur and distal tibia rotations were not found to be different between groups. All four CT rotational measures showed strong repeatability with ICC values ranging from $0.86-0.96$.

Subjects with medial knee OA and tibial intorsion deformity walked with significantly greater moment of support compared to control but not compared to subjects with medial knee OA without the torsion deformity (Table 2, Figure 3). TKO also had significantly greater internal knee extensor moment when compared to $\mathrm{CON}$ but not compared to KOA, though this comparison approached significance (Table 2, Figure 3). No between-group differences were seen for the internal hip and ankle joint moments. It is important to note that the test for between-group differences of the extensor moment values used gait velocity as a covariate to statistically adjust the group means.

\section{Discussion}

Previous studies have quantified the impact of external tibial torsion and surgical intervention on the actual and simulated kinetics of crouch gait for children with cerebral palsy and high sacral myelomeningocele [10-13]. Though a relationship between tibial intorsion and knee 
Table 1. Mean (SD) computed tomography measures of torsion for medial knee osteoarthritis subjects with torsion (TKO) and without (KOA) as compared to aged-matched controls (CON). Inter-rater reliability for the imaging measures estimated by the intraclass correlation coefficient (ICC).

\begin{tabular}{ccccc}
\hline & TKO & KOA & CON & ICC \\
\hline Proximal femur (Degree) & $10.63(9.96)$ & $9.88(6.30)$ & $5.82(6.10)$ & 0.856 \\
Distal femur (Degree) & $9.23^{* \#}(5.73)$ & $-1.86(8.62)$ & $-1.27(7.36)$ & 0.964 \\
Proximal tibia (Degree) & $19.53^{* \#}(8.03)$ & $0.91(9.92)$ & $-0.16(5.44)$ & 0.941 \\
Distal tibia (Degree) & $38.83(5.53)$ & $26.72(11.02)$ & $30.96(9.10)$ & 0.962 \\
\hline
\end{tabular}

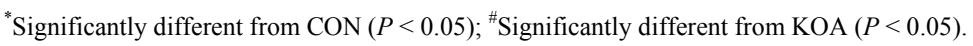

Table 2. The estimated means (SD) when controlling for gait velocity at the first peak vertical ground reaction force for the internal sagittal moment of support, hip, knee and ankle moments of patients with medial knee osteoarthritis and torsion deformity (TKO), patients with medial knee OA (KOA) and healthy controls (CON).

\begin{tabular}{cccc}
\hline & TKO & KOA & CON \\
\hline $\begin{array}{c}\text { Moment of Support } \\
(\% B W B H)\end{array}$ & $8.90^{*}(0.79)$ & $6.86(0.57)$ & $5.50(0.57)$ \\
$\begin{array}{c}\text { Hip Moment } \\
(\% B W B H)\end{array}$ & $3.24(0.51)$ & $2.97(0.37)$ & $2.42(0.37)$ \\
$\begin{array}{c}\text { Knee Moment } \\
(\% B W B H)\end{array}$ & $2.57^{*}(0.42)$ & $1.09(0.30)$ & $1.04(0.30)$ \\
$\begin{array}{c}\text { Ankle Moment } \\
(\% B W B H)\end{array}$ & $3.10(0.53)$ & $2.81(0.38)$ & $2.03(0.38)$ \\
\hline
\end{tabular}

Standard deviations are in parentheses; "Significantly different from CON $(P<0.05)$.

OA has been reported [14,15], few studies have reported the impact of tibial intorsion deformity on gait kinetics $[4,16]$.

This study reports the effect of torsion deformities and knee OA on the extensor moments of the lower extremity during the loading response of gait. The TKO subjects of this study were found to have greater tibial intorsion which was a result of significantly greater external torsion of the proximal tibia, as no between-group difference was seen for the distal tibia rotation. One may expect extensor moment deficits at the hip and knee for TKO due to the impact of the torsion deformity on the insertion sites of the extensor musculature. However, when supporting the body weight on the involved limb, TKO produced significantly greater relative moment of support and internal knee extensor moment compared to CON. This finding was unexpected, but is supported by the findings of Hicks et al. (2007) who report that moment arm reductions are secondary to inter-segmental forces when accounting for diminished extensor muscle capacity in the presence of simulated tibial torsion during gait [6]. An interrupted extensor moment synergy has been reported to occur in the presence of a stiff knee gait pattern for me- dial knee OA subjects where the knee is fixed in flexion across stance phase $[17,18]$. It is possible that the TKO extensor

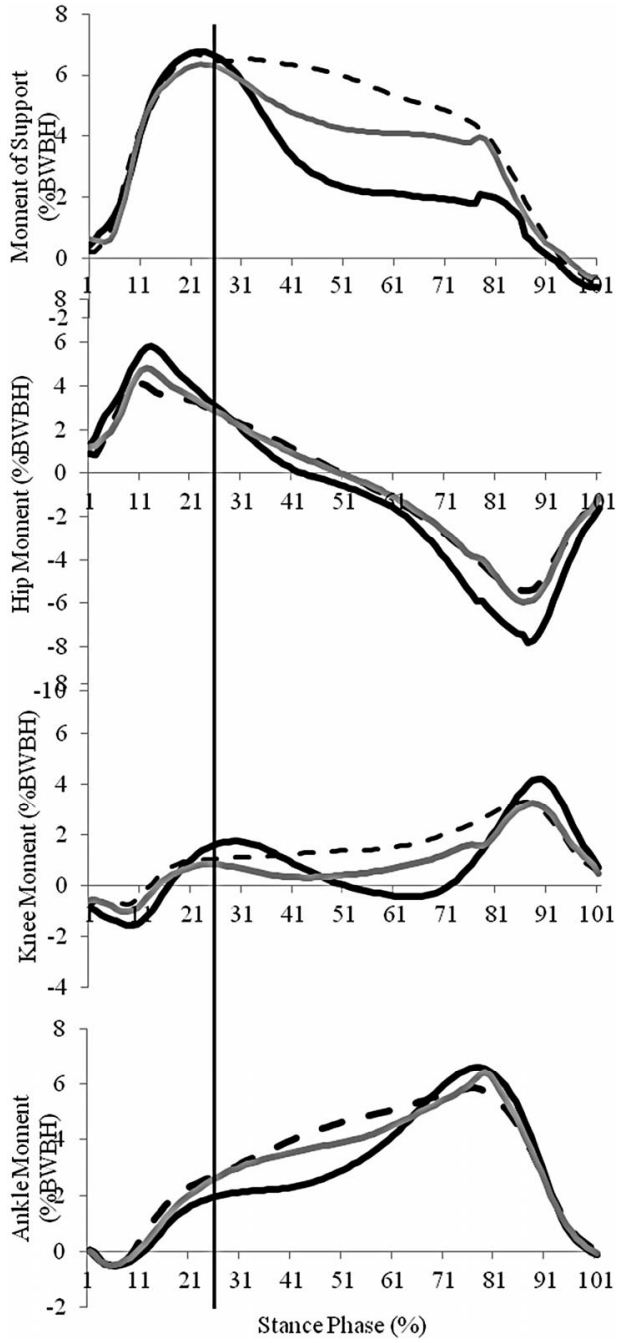

Figure 3. Ensemble curves of internal moment of support and net sagittal joint moments for TKO (dashed), KOA (gray) and CON (black). Values were isolated at the first peak vertical ground reaction force (vertical bar, $25 \%$ stance phase).

moment synergy required increased total moment of support to generate sufficient intersegmental forces when supporting the body weight.

The finding that the KOA subjects without the torsion 
deformity showed similar values of distal femur and proximal tibia rotation compared to $\mathrm{CON}$ that suggests the torsion deformity is not a consequence of the pathologic loading of medial knee OA. It may be proved that progressive genu varum as a result of medial compartment sclerosis overlies an idiopathic pre-existing morphologic intorsion deformity. As conservative interventions of tibial torsion deformity have been shown to be ineffective [11], derotation osteotomy has been suggested to spare future knee OA development in subjects with tibial torsion deformity [15]. Future prospective imaging studies are needed to assess for the presence of independent torsion deformity for initial staged knee OA subjects.

The tibial intorsion values reported in this study showed strong inter-rater reliability and were in the range of those previously reported for subjects with medial knee OA [14.15] and for subjects with rotational malalignment [16]. The CT torsion measures, including those of the proximal tibia, have previously been shown to be robust to differences in slice location and reference axis selection $[19,20]$. However, there is a paucity of evidence regarding the morphology of tibial torsion deformity, though it is commonly believed to occur distally [6]. Our results indicate that the tibial intorsion leads to an externally rotated hip joint with the knee pointing outwards when the patient walks with a normal foot progression angle.

Both the kinetic and torsion results of this study need to be interpreted cautiously due to a limited sample. Future epidemiologic studies are needed to characterize this possible risk factor for medial knee OA. A limitation of this study is that individual muscle contributions and the neurologic input cannot be described for the altered skeletal geometry of tibial intorsion deformity. Future studies employing electromyography are required to confirm increased muscular demand on those muscles with altered mechanical advantage. Though CT torsion measures have been shown to correlate to the transverse plane kinematics from gait analysis [16], these rotation values from gait analysis have been shown to be unreliable [21] and have not been presented here. Future gait studies reporting these variables at the hip and knee might use an a priori Cardan transformation sequence prioritizing the transverse plane and sacrificing the sagittal and frontal planes.

In conclusion, subjects with medial knee OA and tibial intorsion deformity showed greater external rotation of the proximal tibia and distal femur compared to medial knee OA subjects and controls. Thus, the intorsion deformity occurred as a result of a proximal malrotation of the tibia. When controlling for gait velocity, the adjusted TKO values showed greater relative moment of support and extensor moment at the knee. Increased extensor output from TKO may be the result of an increased mus- cular response needed to overcome an interrupted intersegmental exchange of accelerations at the knee.

\section{REFERENCES}

[1] K. Krackow, C. L. Pepe and E. J. Galloway, "A Mathematical Analysis of the Effect of Flexion and Rotation on Apparent Varus/Valgus Alignment at the Knee," Orthopedics, Vol. 13, No. 8, 1990, pp. 861-868.

[2] K. Krackow, "The Technique of Total Knee Arthroplasty," C.V. Mosby Company, Baltimore, 1990.

[3] K. Krackow, C. D. Miller, W. M. Mihalko, L. J. Serpe, M. B. Thering and M. J. Philips, "Thinking about Deformity and Alignment in TKA," Orthopedics, Vol. 20, No. 9, 1997, pp. 825-826.

[4] K. Krackow, D. S. Mandeville, S. R. Rachala, M. Bayers-Thering and L. R. Osternig, "Torsion Deformity and Joint Loading for Medial Knee Osteoarthritis," Gait \& Posture, Vol. 33, No. 4, 2011, pp. 625-629. doi:10.1016/j.gaitpost.2011.02.006

[5] J. Weidow, I. Mars and J. Karrholm, "Medial and Lateral Osteoarthritis of the Knee Is Related to Variations of Hip and Pelvic Anatomy," Osteoarthritis and Cartilage, Vol. 13, No. 6, 2005, pp. 471-477. doi:10.1016/j.joca.2005.01.009

[6] J. Hicks, A. Arnold, F. Anderson, M. Schwartz and S. Delp, "The Effect of Excessive Tibial Torsion on the Capacity of Muscles to Extend the Hip and Knee during Single-Limb Stance," Gait \& Posture, Vol. 26, No. 4, 2007, pp. 546-552. doi:10.1016/j.gaitpost.2006.12.003

[7] D. Mandeville, L. R. Osternig and L. S. Chou, "The Effect of Total Knee Replacement on Dynamic Support of the Body during Walking and Stair Ascent," Clinical Biomechanics, Vol. 22, No. 7, 2007, pp. 787-794. doi:10.1016/j.clinbiomech.2007.04.002

[8] D. Winter, "Overall Principle of Lower Limb Support during Stance Phase of Gait," Journal of Biomechanics, Vol. 13, No. 11, 1980, pp. 923-927. doi:10.1016/0021-9290(80)90162-1

[9] F. Anderson and M. G. Pandy, "Individual Muscle Contributions to Support in Normal Walking," Gait \& Posture, Vol. 17, No. 12, 2003, pp. 159-169. doi:10.1016/S0966-6362(02)00073-5

[10] R. Dunteman, S. J. Vankoski and L. S. Dias, "Internal Derotation Osteotomy of the Tibia: Pre and Postoperative Gait Analysis in Persons with High Sacral Myelomeningocele," Journal of Pediatric Orthopaedics, Vol. 20, No. 5, 2000, pp. 623-628. doi:10.1097/01241398-200009000-00014

[11] R. Stefko, R. J. de Swart, D. A. Dodgin, M. P. Wyastt, K. R. Kaufman, D. H. Sutherland and H. G. Chambers, "Kinematic and Kinetic Analysis of Distal Derotational Osteotomy of the Leg in Children with Cerebral Palsy," Journal of Pediatric Orthopaedics, Vol. 18, No. 1, 1998, pp. 81-87. doi:10.1097/01241398-199801000-00016

[12] A. Arnold, S. S. Blemker and S. L. Delp, "Evaluation of a Deformable Musculoskeletal Model for Estimating Muscle-Tendon Lengths during Crouch Gait," Annals of Bio- 
medical Engineering, Vol. 29, No. 3, 2001, pp. 263-274. doi:10.1114/1.1355277

[13] S. Ounpuu, P. DeLuca, R. Davis and M. Romness, "Long-Term Effects of Femoral Derotation Osteotomies: An Evaluation Using Three-Dimensional Gait Analysis," Journal of Pediatric Orthopaedics, Vol. 22, No. 2, 2002, pp. 139-145. doi:10.1097/01241398-200203000-00001

[14] M. Turner, "The Association between Tibial Torsion and Knee Joint Pathology," Clinical Orthopaedics and Related Research, Vol. 302, 1994, pp. 47-51.

[15] J. Weidow, R. Tranberg, T. Saari and J. Karrholm, "Hip and Knee Joint Rotations Differ between Patients with Medial and Lateral Knee Osteoarthritis: Gait Analysis of 30 Patients and 15 Controls," Journal of Orthopaedic Research, Vol. 24, No. 9, 2006, pp. 1890-1899. doi:10.1002/jor.20194

[16] C. Radler, A. Kranzl, H. M. Manner and M. Hoglinger, "Torsional Profile versus Gait Analysis: Consistency between the Anatomic Torsion and the Resulting Gait Pattern in Patients with Rotational Malalignment of the Lower Extremity," Gait \& Posture, Vol. 32, No. 3, 2010, pp. 405-410. doi:10.1016/j.gaitpost.2010.06.019
[17] D. Mandeville, L. R. Osternig and L. S. Chou, "The Associations between Knee Flexion, Vertical Center-of-Mass Excursions and Lower-Extremity Muscle Work for EndStage Knee Osteoarthritis," Journal of Musculoskeletal Research, Vol. 12, No. 2, 2009, p. 7. doi:10.1142/S0218957709002213

[18] C. McGibbons and D. E. Krebs, "Compensatory Gait Mechanics in Patients with Unilateral Knee Arthritis," Journal of Rheumatology, Vol. 29, No. 11, 2002, pp. 2410-2419.

[19] D. Hudson, T. Royer and J. Richards, "Ultrasound Measurements of Torsions in the Tibia and Femur," The Journal of Bone \& Joint Surgery, Vol. 88, No. 1, 2006. pp. 138-143. doi:10.2106/JBJS.D.02924

[20] D. Eckhoff, "Effect of Limb Malrotation on Malalignment and Osteoarthritis," Orthopedic Clinics of North America, Vol. 25, No. 3, 1994, pp. 405-414.

[21] M. Kadaba, H. K. Ramakrishnan and M. E. Wooten, "Measurement of Lower Extremity Kinematics during Level Walking," Journal of Orthopaedic Research, Vol. 8, No. 3, 1990, pp. 383-392. doi:10.1002/jor.1100080310 